\title{
Diagnosis, management, and long-term outcomes of rectovaginal endometriosis
}

\author{
This article was published in the following Dove Press journal: \\ International Journal of Women's Health \\ 7 November 2013 \\ Number of times this article has been viewed
}

\author{
Nash S Moawad' \\ Andrea Caplin² \\ 'Minimally Invasive Gynecologic \\ Surgery, Department of Obstetrics \\ and Gynecology, University of Florida, \\ ${ }^{2}$ University of Florida College of \\ Medicine, Gainesville, FL, USA
}

Correspondence: Nash S Moawad Department of Obstetrics and Gynecology, University of Florida College of Medicine, PO Box 100294 ,

Gainesville, FL, USA 32610-0294

Tel + I 3522737660

Fax + I 3523923498

Email nmoawad@ufl.edu

\begin{abstract}
Rectovaginal endometriosis is the most severe form of endometriosis. Clinically, it presents with a number of symptoms including chronic pelvic pain, dysmenorrhea, deep dyspareunia, dyschezia, and rectal bleeding. The gold standard for diagnosis is laparoscopy with histological confirmation; however, there are a number of options for presurgical diagnosis, including clinical examination, transvaginal/transrectal ultrasound, magnetic resonance imagining, colonoscopy, and computed tomography colonography. Treatment can be medical or surgical. Medical therapies include birth control pills, oral progestins, gonadotropin-releasing hormone agonists, danazol, and injectable progestins. Analgesics are often used as well. Surgery improves up to $70 \%$ of symptoms. Surgery is either ablative or excisional, and is conducted via transvaginal, laparoscopic, laparotomy, or combined approaches. Common surgical techniques involve shaving of the superficial rectal lesion, laparoscopic anterior discoid resection, and low anterior bowel resection and reanastomosis. Outcomes are generally favorable, but postoperative complications may include intra-abdominal bleeding, anastomotic leaks, rectovaginal fistulas, strictures, chronic constipation, and the need for reoperation. Recurrence of rectal endometriosis is a possibility as well. Other outcomes are improved pain-related symptoms and fertility. Long-term outcomes vary according to the management strategy used. This review will provide the most recent approaches and techniques for the diagnosis and treatment of rectovaginal endometriosis.
\end{abstract}

Keywords: pelvic pain, dyspareunia, bowel resection, endometriosis, rectovaginal

\section{Introduction}

Rectovaginal endometriosis (RVE) is one of the most severe forms of endometriosis, and is considered stage 4 according to Kirtner's classification. ${ }^{1,2}$ It is much less common than ovarian or peritoneal endometriosis and affects between $3.8 \%$ and $37 \%$ of all patients with endometriosis. ${ }^{3,4}$ RVE is deep infiltrating endometriosis (DIE) that infiltrates the vagina, rectum, and the rectovaginal septum, and it obliterates the posterior cul-de-sac or the pouch of Douglas. ${ }^{4}$ Furthermore, in cases where the endometriotic nodule exceeds $30 \mathrm{~mm}$ in diameter, ureteral involvement occurs in $17.9 \%$ of patients. ${ }^{5}$ Anywhere from $5.3 \%-12 \%$ of patients are estimated to have bowel endometriosis. The rectosigmoid is the most common site of gastrointestinal involvement affecting $74 \%$ of those patients. 3,4

Preoperative diagnosis can be challenging. There is a notable absence of agreed upon disease-specific endoscopic and radiological features. However, several diagnostic methods have been proposed and studied in the literature including digital rectovaginal examination, transvaginal/transrectal ultrasounds, magnetic resonance 
imaging (MRI), colonoscopy, computed tomography (CT) colonography and, ultimately, laparoscopic excision with histological confirmation. ${ }^{6-8}$

In RVE, medical treatments can be used, but they are often ineffective or only temporarily effective in controlling the associated symptoms. Complete removal of the endometriotic tissue is what provides the most long-term pain relief and improved quality of life. ${ }^{4}$ Thus, surgery is the definitive treatment of choice for most women. ${ }^{1,4}$ Surgery is either ablative or excisional, and is conducted via transvaginal, laparoscopic, laparotomy, or combined approaches. ${ }^{4}$ Common surgical techniques involve shaving of the superficial rectal lesion, laparoscopic anterior discoid resection (ADR), and low anterior bowel resection and reanastomosis. ${ }^{1}$

Surgical therapy of bowel endometriosis can be technically challenging and lengthy. When bowel surgery is necessary for treatment, complications increase up to $53 \%{ }^{4}$ Bowel resections occur needlessly in $1.7 \%-28.6 \%$ of cases. ${ }^{4}$

It is clear that RVE has a number of diagnostic and management options. Long-term outcomes vary according to the management strategy used. This review will provide the most recent approaches and techniques for the diagnosis and treatment of RVE.

\section{Methods}

This review aims to describe the state-of-the-art diagnostic and treatment modalities of RVE. The database PubMed (National Center for Biotechnology Information, US National Library of Medicine, Bethesda, MD, USA) was used. The terms used most frequently in the search were: "recto-sigmoid endometriosis, recto-vaginal endometriosis, deep infiltrating endometriosis, and a combinations of these terms with the terms pelvic pain, dyspareunia, ovarian endometrioma, transvaginal ultrasound, trans-vaginal sonography, trans-rectal ultrasound, trans-rectal sonography, colonoscopy, MRI, CT, laparoscopy, anterior discoid resection, segmental bowel resection, and quality of life". No restrictions were used. If the article was published in another language, the information would still be used if an English abstract was available. The search was limited to primarily the last 10 years, although most of the articles cited were published within the last 5 years. References from articles were reviewed and pertinent articles were used.

\section{Clinical presentation}

Symptoms of DIE include chronic pelvic pain, dysmenorrhea, deep dyspareunia, and dyschezia. ${ }^{1}$ Endometriosis infiltrating the lumen of the intestine can cause obstruction
(Figure 4), rectal bleeding, hemorrhagic ascites, protein loss, intussusception, and edema. ${ }^{9}$ The degree of pain in relation to the severity of the condition varies among patients. Some cases of mild endometriosis can be associated with significant pain and other cases with severe endometriosis may experience little or no pain. Some estimate that $5 \%$ of patients with DIE are pain-free. ${ }^{3,10}$ Pain can be caused by compression or infiltration of specific nerves by the ectopic endometrial growth, or the anatomical position of the lesion on the organ can prevent or interfere with its function. ${ }^{11}$ Histological evaluation of RVE with severe pain is associated with a higher proportion of intraneural and perineural infiltration. Nerves were shown to be in close relationship with the endometriotic nodules and the fibrotic tissue, and this was suggestive of the relationship between endometriotic lesions and pain. ${ }^{12}$ Pain variations can be attributed to several factors.

The cytokines and growth factors (estradiol, prostaglandins, and nerve growth factor) associated with endometriosis have been correlated with pain sensation. In addition, current studies have proposed that endometriosis causes a hyperalgesic state whereby endometriotic lesions could stimulate peripheral nerve fibers to sensitize the central nervous system and lead to phantom pains in the absence of lesions. ${ }^{13}$ This could impact treatment strategies and help explain the varying relief patients report experiencing from therapy. The variation in symptoms can make the disease challenging to identify clinically.

Dyspareunia is a common symptom of patients with DIE. In an article published in 2013 from the International Society for Sexual Medicine, ${ }^{14}$ the Sexual Health Outcomes in Women Questionnaire and the Short Form-36 questionnaire evaluating quality of life analyzed the sexual impairment and related quality of life of patients with DIE. The results showed a decrease in sexual satisfaction and sense of well-being. Other analyses showed that RVE had a $67 \%$ dyspareunia rate in comparison with a $53 \%$ dyspareunia rate among women with endometriosis in other regions, such as in the peritoneum or ovaries. These differences were considered marginal and could be indicative of equal sexual dissatisfaction among patients with all types of endometriosis. ${ }^{15}$

Some patients with DIE may have problems with fertility and seek reproductive therapy such as in vitro fertilization (IVF). Studies have shown that those patients with this severe form of endometriosis have a lower cumulative pregnancy rate in comparison with patients with less severe forms of endometriosis. ${ }^{16}$ Those with DIE have a cumulative pregnancy rate of $69.4 \%$, which can increase slightly after several rounds of in vitro fertilization. However, after three rounds of IVF, 
surgery is suggested. ${ }^{16}$ Data from other studies revealed no difference in pregnancy rates when comparing patients with different stages of endometriosis. ${ }^{17}$ The only difference found was an increase in the interval between surgery and pregnancy among patients with DIE.

\section{Risk factors for deep infiltrating endometriosis}

The group most affected by DIE includes those who fall in the age group of $21-25$ years. ${ }^{18}$ About $20 \%$ of women develop severe endometriosis as either the deep infiltrating type, or as cystic ovarian disease.$^{19}$ There is a significant delay between the onset of disease and diagnosis (about 10 years). In addition, about $74 \%$ of patients receive one false diagnosis before being accurately diagnosed. ${ }^{20}$

Several markers or clues that point to the diagnosis of DIE can be identified by taking a thorough history. A family history of endometriosis is a primary risk factor. In fact, a range of data reports that there is a two- to tenfold risk of developing endometriosis when a first-degree relative has the disease. ${ }^{13,21,22}$ Other risk factors include absenteeism from school during menstruation and early and prolonged use of oral contraceptives for the treatment of primary dysmenorrhea. Nonsteroidal anti-inflammatory drugs are the first line of therapy used for primary dysmenorrheal, and oral contraceptive pills (OCPs) are generally the next choice. However, for those women with DIE, birth control pills are generally insufficient therapy. ${ }^{22}$

A cross-sectional study found that the use of oral contraceptives for primary dysmenorrhea is associated with a surgical diagnosis of DIE, with an adjusted odds ratio of 16.2. This is likely not a causative relationship, but rather, the need for the long-term use of OCPs can be used as a marker for the development of DIE. ${ }^{23}$ In addition, using combined oral contraceptives preoperatively has been associated with a decreased need for bowel resection. However, the temporary symptom control that oral contraceptives provide might have impacted surgical decision making. Such symptom control includes relieving endometriosis-related dysmenorrhea and preventing the recurrence of ovarian endometriomas. Evidently, more research is needed to assess the effects of hormonal therapies in the management of RVE. ${ }^{3}$

Ovarian endometriomas have been considered markers for severe and multifocal DIE. ${ }^{24}$ Ovarian endometriomas are known to coexist with deep infiltrating endometriotic lesions affecting the uterine surface, cul-de-sac, and the uterosacral ligaments. ${ }^{25}$ In a study evaluating 153 patients undergoing resection for RVE, one-third of patients had concomitant endometriomas. ${ }^{3}$ Thus, it is important to ensure that all endometriotic lesions are removed during surgery to maximize the relief of pelvic pain symptoms and to minimize the risk of recurrence. ${ }^{25} \mathrm{In}$ addition, infertility is a long-term outcome that results from endometriomas that adhere to the ovaries or fallopian tubes.

\section{Diagnosis}

Common presenting symptoms of RVE include dysmenorrhea, dyschezia, dyspareunia, chronic pelvic pain, and bowel symptoms including rectal bleeding, bloating, diarrhea, and constipation. ${ }^{1,26}$ In $80 \%$ of patients with RVE, a triad of dysmenorrhea, bowel ailments, and dyspareunia present upon diagnosis. ${ }^{1}$ Some studies report that digestive symptoms are not usually indicative of the endometriotic lesion location. ${ }^{27}$ The symptoms are generally caused by the inflammatory factors irritating the bowel, rather than by infiltration of the disease into the bowel - except in a few cases where stenosis of the bowel lumen results from the associated fibrosis. ${ }^{27}$ However, other analyses have revealed that some types of pain are indicative of location. Findings showed that dysmenorrhea increased with pouch of Douglas infiltration, dyspareunia increased with uterosacral ligament infiltration, noncyclic pelvic pain increased with bowel involvement, dyschezia during menstruation increased with vaginal infiltration, lower urinary tract symptoms increased with bladder involvement, and gastrointestinal symptoms were associated with bowel and vaginal involvement. ${ }^{28}$

During a digital rectovaginal exam, a mass in the rectovaginal or rectocervical region can sometimes be detected. Generally, a nodule must be palpated and found to be painful in the cul-de-sac or uterosacral region in order for it to be diagnostically relevant. ${ }^{6}$ Research has shown that in those regions, the positive predictive value (PPV) of diagnosing endometriosis clinically by tenderness, nodularity, tenderness with nodularity, and tenderness or nodularity is $85.5 \%, 94 \%$, $94.6 \%$, and $86.7 \%$, respectively. ${ }^{29}$ In another study, digital vaginal examination in general had a sensitivity of $72 \%$, specificity of $54 \%$, PPV of $63 \%$, negative predictive value (NPV) of $68 \%$, and an accuracy of $63 \%$ for the detection of a rectovaginal lesion. ${ }^{6}$ In addition, this procedure had a sensitivity of $68 \%$, specificity of $46 \%$, PPV of $45 \%$, NPV of $69 \%$, and an accuracy of $55 \%$ for the detection of a rectocervical lesion. Although an essential element of the initial assessment, a physical examination is generally of limited value in evaluating DIE because of its poor ability to discriminate whether growths involve the vagina, rectovaginal space, or uterosacral ligaments. ${ }^{30}$ 
Laparoscopy is the gold standard for the diagnosis of endometriosis, and histological confirmation can be beneficial due to high false-positive rates of visual diagnosis. Due to the invasiveness of the procedure, other methods are often employed to detect the lesion and to aid with preoperative planning and patient counseling. Transvaginal ultrasound (TVS), transrectal ultrasound, CT colonography, and MRI are examples of the preoperative methods available to detect deep infiltrating RVE. ${ }^{6}$ There is varying data on which offers the highest sensitivity, specificity, PPV, NPV, and accuracy in cases of deep RVE. According to one study, TVS was superior to other modalities in detecting RVE, with a sensitivity of $98 \%$, specificity of $100 \%$, PPV of $100 \%$, and NPV at $98 \%{ }^{6}$ In contrast, the study found MRI to have values of $83 \%, 98 \%, 98 \%$, and $85 \%$ respectively. Other studies have shown similar results with sensitivities of $73.9 \%-98 \%$, and specificities of $87.5 \%-100 \%$ with TVS. ${ }^{31-34}$ In a cohort study consisting of 422 patients, sensitivities and specificities of TVS were categorized according to the rectovaginal septum, rectum, and sigmoid colon. Results were 52\% and $96 \%, 65 \%$ and $99 \%$, and $69 \%$ and $98 \%$, respectively. ${ }^{35}$ There has been data collected on the high degree of false negatives that TVS generates at the uterosacral ligament and when the pouch of Douglas is obliterated (Figure 1). ${ }^{35}$

According to a study published in 2012, TVS accompanied by the use of saline injection (saline contrast sonovaginography [SCSV]) showed superior sensitivity, specificity, PPV, and NPV. It is believed that the pressure from the saline solution creates a clearer view of the walls of the vagina. It can thus detect the location and invasion of the lesions more accurately. ${ }^{36}$ In addition, patients in this study were more comfortable with this technique, though the data

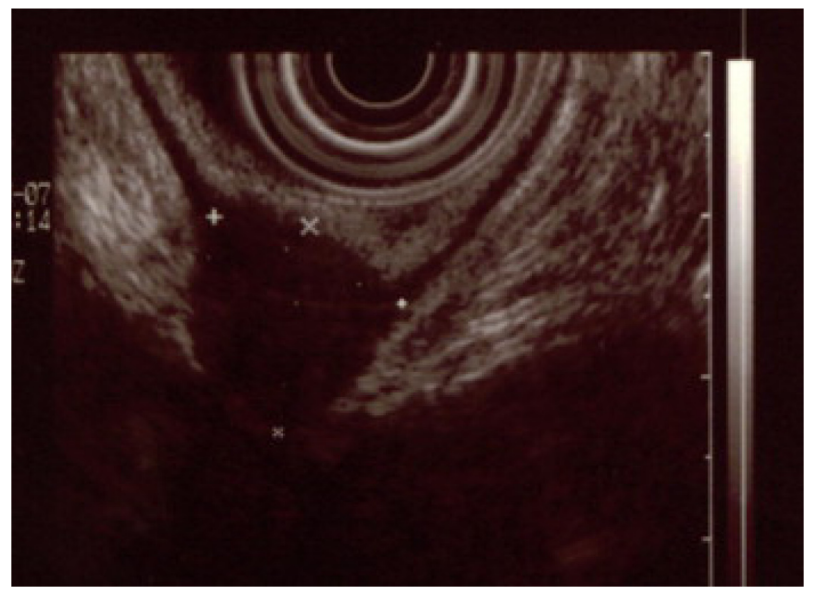

Figure I This is an endorectal ultrasound revealing a deep endometriosis nodule involving the muscular layer of the rectum.

Note: Copyright (C) 2013. Reproduced with permission of Elsevier. Roman H, Vassilieff M, Tuech JJ, et al. Postoperative digestive function after radical versus conservative surgical philosophy for deep endometriosis infiltrating the rectum. Fertil Steril. 2013;99(6): 1695-1704. ${ }^{62}$ for this finding were not statistically significant. Although SCSV appeared superior to traditional TVS in this study, the authors did not suggest its use as a first-line strategy unless the physician had extensive training in this approach. In the analysis, MRI had the same results as SCSV in diagnosing rectal endometriosis with matching sensitivities of $66.7 \%$. This value supersedes the $33.3 \%$ sensitivity found with the use of TVS alone in this study. Although MRI sensitivity is quantitatively superior to TVS in this analysis, it can be very time consuming and quite costly. SCSV could be a more sensitive and affordable option if physicians are adequately trained. ${ }^{36}$

In a study from $2003,{ }^{37} \mathrm{SCSV}$ presented with similar data to those discussed above. The results revealed TVS to have a sensitivity of $90.6 \%$, specificity of $85.7 \%$, PPV of $93.5 \%$, and NPV of $80 \%$. In addition, SCSV was associated with minimal pain and discomfort.

Another variation of TVS includes the "tenderness guided" approach, which uses excess amounts of gel to create an acoustic window of pressure to guide the physician to areas where the patient exhibits more pain, thus identifying the areas of endometriotic tissue. This technique found specificities of $95 \%$ and sensitivities of $90 \%$, suggesting another inexpensive yet sensitive approach to diagnosis. ${ }^{38}$

The uterine sliding sign is a useful diagnostic tool that can be employed during TVS. This technique presses the transducer into the posterior vaginal fornix and withdraws backwards to determine the motion of the rectum against the posterior vaginal fornix and posterior uterine wall in the midsagittal plane. External pressure is also applied to the uterus by placing one's hand on top of the abdomen. If movement is absent, the uterine sliding sign is considered negative and is suggestive of adhesions. ${ }^{30}$ In a study that evaluated this approach in regards to DIE located in the pouch of Douglas, a negative uterine sliding sign was indicative of endometriosis with a sensitivity of $83.3 \%$, specificity of $97.1 \%$, PPV of $92.6 \%$, NPV of $93.2 \%$, and an accuracy of $93 \%$. This approach is considered useful in women with a high risk for bowel endometriosis because of its high degree of certainty, especially with regards to the involvement of the pouch of Douglas. ${ }^{39}$

Both transvaginal and transrectal ultrasound can detect endometriosis as an irregular mass that is hypoechoic with a hyperechoic rim. Transrectal ultrasounds, however, can be quite useful in measuring the distance from the endometriotic lesion to the anal verge. In fact, in a retrospective study, ${ }^{40}$ rectal endoscopic sonography showed a PPV of 100\% in detecting muscularis layer infiltration; however, it was not 
as accurate in determining infiltration of the submucosal/ mucosal layers. Sensitivity, specificity, PPV, and NPV for those layers were $89 \%, 26 \%, 55 \%$, and $71 \%$, respectively. It is important to note that there have been results where specificity in the submucosa/mucosa is as high as $94 \% .{ }^{34}$

Colonoscopy can be used to diagnose endometriosis that has invaded into the bowel as well. ${ }^{8}$ Rectoscopy is not commonly used for lesions because growths generally do not penetrate past the muscularis propria $(<10 \%)$ or mucosa. Thus, colonic endometriomas are easily missed on colonoscopy. ${ }^{7,8}$ Because of the clinical symptoms and endoscopic/radiologic findings, RVE can be mistaken for malignancy, and thus must be considered in the differential diagnosis when a patient is undergoing colonoscopy. ${ }^{8}$

CT-based virtual colonoscopy is useful in determining stenosis of the bowel due to the infiltration of endometriosis. It is particularly efficient in evaluating whether the patient is in need of shaving or full-thickness resection when presenting with DIE in the colon. ${ }^{41}$

Double-contrast barium enemas have an accuracy of $90 \%$ and PPV of $97 \%$ in diagnosing colonic involvement of endometriosis. ${ }^{35}$

Modified virtual colonoscopy, also known as CT colonography, is another useful tool for determining the infiltration of endometriosis into the bowel. CT colonography has a wide range of views expanding into the submucosa and the serosa, unlike traditional colonoscopy. In this modified approach, a large obstetric tampon is inserted high into the vagina, a Foley catheter is inserted into the rectum releasing $\mathrm{CO}_{2}$, and the pelvis is scanned. This technique is noninvasive, of short duration (20 minutes), requires minimal preparation, and does not require sedation. It uses a lower dose of radiation than virtual colonoscopy, pyelogram, and barium enemas. No endoscope or barium is required, and it is approximately $50 \%$ less costly than optical colonoscopy. The main advantage is that the bowel is insufflated with $\mathrm{CO}_{2}$, which allows for multiple viewing modalities. Abdominal, retroperitoneal, and pelvic organs can be scanned as well. DIE can be visualized in the bowel, urinary tract, as well as in the rectovaginal and recto-peritoneal regions. This is a good technique for young women who wish to avoid high radiation doses and need a comprehensive presurgical evaluation because it allows for almost all presurgical investigation to be completed in one study (Figure 2). ${ }^{42,43}$

A descriptive imaging classification was proposed using modified virtual colonoscopy in an attempt to quantify the severity of rectogenital disease and disseminated endometriosis. The LSD/MURO classification signifies the severity of the pathology by giving it a numeric evaluation

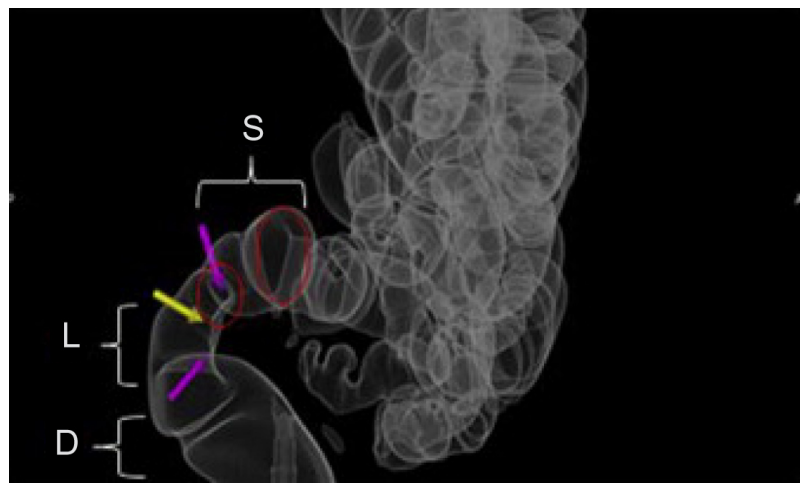

Figure $\mathbf{2}$ This is a modified virtual colonography with transparent views of the rectum and lower sigmoid.

Notes: The strictured area in the rectogenital area is indicated by arrows. The L, S, and $D$ in the figure are from the LSD/MURO classification system. Copyright @ 2013. Reproduced with permission of Elsevier. van der Wat J, Kaplan MD, Roman H, Da Costa $\mathrm{C}$. The use of modified virtual colonoscopy to structure a descriptive imaging classification with implied severity for rectogenital and disseminated endometriosis. J Minim Invasive Gynecol. Epub June 5, 2013. ${ }^{42}$

Abbreviations: L, length; S, stricture; D, distance to the anal verge; MURO, describes disseminated endometriosis beyond the rectogenital organs.

(ie, $0,1,2$, or 3 ). Rectogenital disease is described by the LSD component; it identifies and quantifies pathology from the anal verge to the lower sigmoid.

L corresponds to the length of stricture $(0=$ no stricture or rectogenital nodule with no bowel involvement; $1=$ a stricture length of $<3 \mathrm{~cm} ; 2=$ a stricture length of $3-5 \mathrm{~cm}$; and $3=\mathrm{a}$ stricture length $>5 \mathrm{~cm}$ and/or non-distensibility). The stricture (S) is calculated by measuring the smallest stricture diameter and comparing it with the closest normal bowel lumen diameter ( $0=$ no stricture; $1=<30 \%$ stricture $; 2=30 \%-60 \%$ stricture; and $3=>60 \%$ stricture). $\mathrm{D}$ is the distance from the anal verge $(0=$ bowel involvement; $1=>15 \mathrm{~cm} ; 2=8-15 \mathrm{~cm}$; and $3=<8 \mathrm{~cm})$. The score increases as the pathology gets closer to the anal verge. MURO describes disseminated endometriosis beyond the rectogenital organs; $M$ corresponds to multifocal disease above the lower sigmoid, $\mathrm{U}$ corresponds to urinary tract involvement, R corresponds to reproductive organ involvement, and $\mathrm{O}$ corresponds to abdominal organs such as the liver.

MRI is often the preoperative, noninvasive method of choice used to diagnose RVE due to its high level of accuracy in predicting specific locations of the infiltrating endometriosis in the intestinal, pelvic, and retroperitoneal regions. , $36,44,45^{3}$ It should also be noted that MRI has an overall specificity of $90.3 \%$ in predicting lesions in the pelvic and retroperitoneal regions. ${ }^{45} \mathrm{MRI}$ allows precise localization of predicted lesions and visualization of multiple planes of multifocal, scattered, and small lesions. ${ }^{36}$ Though the aforementioned studies $3,36,44,45$ stated that MRI was useful in the intestinal, pelvic, and retroperitoneal regions preoperatively, a different study revealed it was particularly helpful in detecting lesions invading the bladder region, and less effective in the peritoneal regions. ${ }^{46}$ 
The extent of disease infiltration is important for selecting which surgical technique will be employed. Nonetheless, during surgical excision, lesions are occasionally underestimated due to their location, or overestimated secondary to the extent of the fibrotic tissue present. ${ }^{47} \mathrm{MRI}$ may be contraindicated or avoided in patients with significant anxiety or claustrophobia, and CT can be a good option for those patients, with sensitivity, specificity, PPV, NPV, and accuracy values of $87 \%$, $100 \%, 100 \%, 77 \%$, and $91 \%$, respectively. It can be especially helpful in detecting the endometriotic bowel implants $(98.7 \%$ specificity) when combined with a water enema. ${ }^{45}$

Specificity on MRI is exceptionally high; however, research has been conducted on techniques that further improve specificity. One such technique included using 100-120 mL of nonsterile ultrasound gel to opacify the vagina and rectum before imaging. ${ }^{48}$ This allowed for delineation of the pelvic organs, especially of the vagina and rectovaginal septum. The sensitivity increased from $63.1 \%$ to $81.7 \%$ after opacification. In addition the specificity, though almost perfect prior to opacification (99.28\%), reached $100 \%$ after opacification. Other techniques involve recognizing a sub center foci and $\mathrm{T} 2$ hyperintensity to visualize ectopic endometrial glands within fibrotic masses (Figure 3). ${ }^{49}$

Another analysis found that different modalities work better for different regions. TVS worked best for

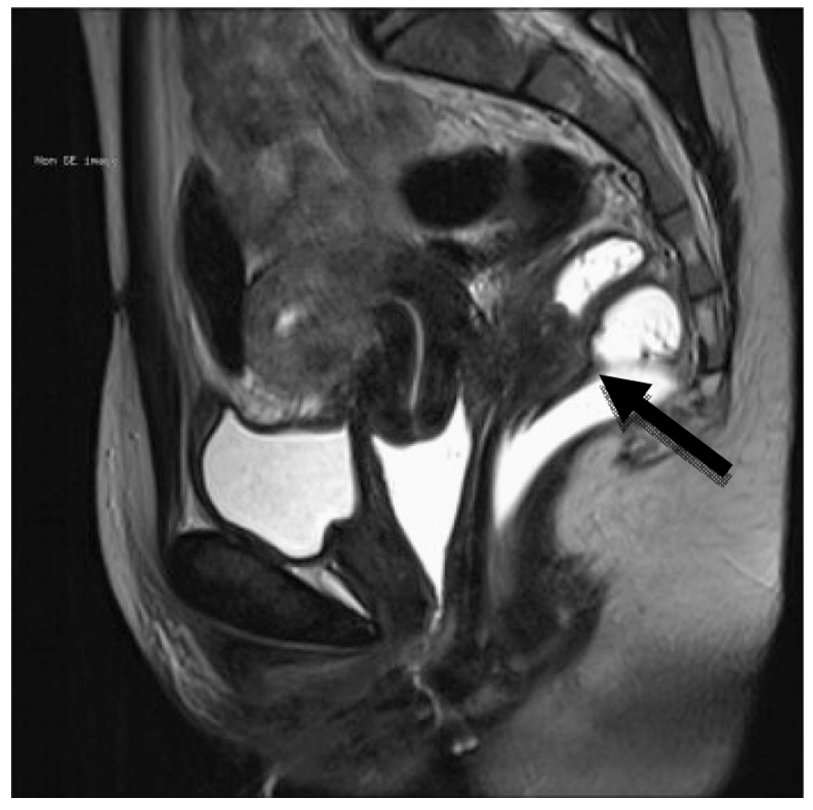

Figure 3 This is a preoperative assessment using magnetic resonance imaging, revealing a deep infiltrating endometriosis nodule with an obvious increase in rectal wall thickness. Notes: The increase in rectal wall thickness is indicated by the arrow. Copyright (C) 2013. Reproduced with permission of Elsevier. Roman H, Vassilieff M, Tuech JJ, et al. Postoperative digestive function after radical versus conservative surgical philosophy for deep endometriosis infiltrating the rectum. Fertil Steril. 2013;99(6):1695-1704. ${ }^{62}$ endometriomas; MRI worked best for uterosacral ligaments, and small lesions on the bladder; and rectal endoscopic sonography worked best for the rectovaginal septum. In conclusion, the study found that all three were complementary for preoperative diagnosis. ${ }^{50}$

\section{Treatment options}

Pain management for RVE is highly variable. There are a number of hormonal therapies, which can partially alleviate pain. These therapies include birth control pills, gonadotropin-releasing hormone agonists, danazol, injectable progestins, and analgesics. ${ }^{13}$ Oral progestins are an option as well. One study revealed both surgical therapy and medical therapy with progestins equally improved deep dyspareunia in women with RVE. ${ }^{51}$ These data are suggestive of the fact that if the patients' only complaints surround dyspareunia, a conservative approach that could be used is hormonal therapy with progestins. Furthermore, low-dose OCPs have shown some benefit in reducing bowel endometriosis-related symptoms of dysmenorrhea, dyspareunia, and dyschezia. In addition to pain relief, therapy with OCPs has been shown to significantly reduce volumetric colorectal plaques when visualized by endoscopic ultrasound. ${ }^{52}$ Also, OCPs have been shown to reduce the risk for bowel resection and the recurrence of ovarian endometriomas. ${ }^{3}$

Although not approved for use in endometriosis by the US Food and Drug Administration, the levonorgestrel-releasing intrauterine system used after conservative surgery can be effective in reducing chronic pain. ${ }^{53}$

Most often, surgery provides the most relief and improves at least $70 \%$ of pain-related symptoms. ${ }^{13,54}$ There are a number

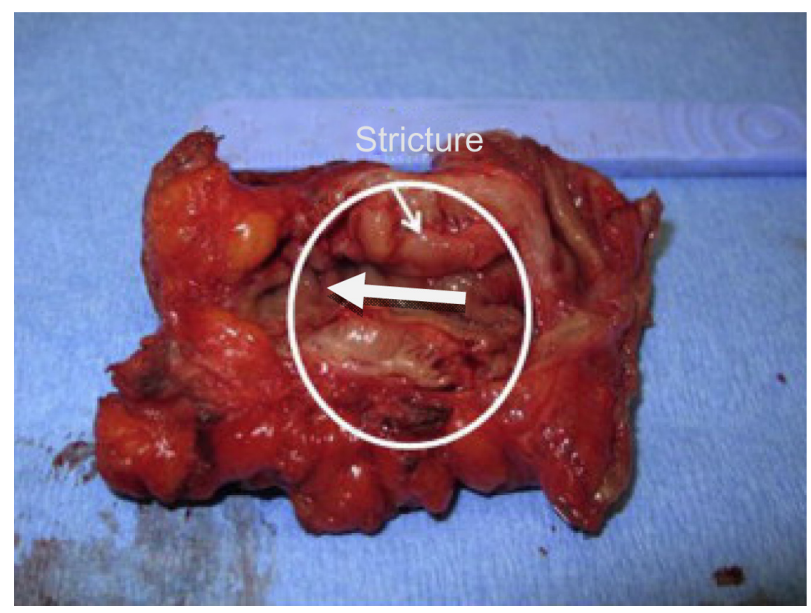

Figure 4 This is an endometriotic stricture $>30 \%$ managed by segmental resection. Note: Copyright (C) 2013. Reproduced with permission of Elsevier. van der Wat J, Kaplan MD, Roman H, Da Costa C. The use of modified virtual colonoscopy to structure a descriptive imaging classification with implied severity for rectogenital and disseminated endometriosis. J Minim Invasive Gynecol. Epub June 5, 2013.42 
of surgical options for patients. Conventional laparoscopic or robotic approaches have been used.$^{55}$ Laparoscopic shaving resections, also known as superficial partial thickness excisions, are used in less severe cases and are associated with fewer complications of bowel obstruction and nerve damage. ${ }^{47,56}$ In a retrospective analysis that examined the various resection techniques of rectal endometriosis, the shaving of superficial lesions had a $6 \%$ complication rate $(\mathrm{CR})$ in comparison with a $23 \% \mathrm{CR}$ in laparoscopic discoid resection and a $38 \% \mathrm{CR}$ with laparoscopic segmental resection. It is important to note that the majority of lesions handled with the shaving technique were smaller than $1 \mathrm{~cm}$; the procedure was less invasive, and thus more easily managed in this manner during surgery. In more extensive cases, segmental and discoid resections were performed. Though complications were less frequent with the shaving approach, pain relief was less effective. This could be due to incomplete excision and recurrence of the endometriotic infiltrate. ${ }^{47}$ Other studies have compared patients treated conservatively to patients treated with radical excision of DIE and have concluded similarly that radical removal greatly decreased the severity of dysmenorrhea and the recurrence of pelvic pain. ${ }^{57}$

In addition, several energy sources can be used in treatment, including laser or electrosurgery ${ }^{58}$ Laparoscopic laser resection is especially helpful in removing infiltrations of the rectovaginal septum and rectovaginal pouch. ${ }^{56,59}$ In a study that used a $\mathrm{CO}_{2}$ laser, out of 48 patients, five needed further surgery over a 17.9-month range, and there was a 19\% recurrence rate. In addition, extreme fatigue reduced from $58 \%$ of patients to $10 \%$ of patients after laser resection. Finally, $59 \%$ of patients considered improvement "excellent", and $41 \%$ considered improvement "satisfactory." 59

Laparoscopic $\mathrm{CO}_{2}$ laser was used in another study that compared women who had laser excision with and without bowel resection. ${ }^{60}$ Clinical outcomes for both groups were equal, except for a higher minor CR occurring in women with bowel resection. Laparoscopic laser ablation, in combination with segmental bowel resection, is associated with good clinical outcomes for women with DIE. ${ }^{61}$

A small retrospective study found that laparoscopic ADR, when feasible, was superior to low anterior resection (LAR) due to decreased operative time, blood loss, and length of hospitalization. In addition, ADR improved symptoms of diarrhea, constipation, daily performance, dyschezia, rectal pain, and dyspareunia more effectively than LAR. These data suggest that ADR - when feasible - should be the initial surgical treatment, except in cases where there is multifocal rectal involvement, large rectal nodules, luminal stenosis, and recurrent disease. In these situations, LAR and reanastomosis may be the patient's best option. ${ }^{1}$ When radical resection was compared with the conservative approach of rectal shaving and nodule excision, the conservative approach had better results on the Knowles-Eccersley-Scott Symptom Questionnaire, Gastrointestinal Quality of Life Index, depression/self-perception Fecal Incontinence Quality of Life score, and significantly improved values for postoperative constipation. Once again, this study suggests better outcomes if the rate of colorectal resection is reduced. ${ }^{62}$

The classical laparoscopic technique is non nerve-sparing and removes parts of the mesentery attached to the posterior colon. This can lead to the damaging of nerves that coordinate bladder and intestinal function, and can result in a loss of vasculature to the bowel. This is often the technique used by colorectal surgeons for colon cancer. However in RVE, a surgeon can use a nerve-sparing technique, which takes advantage of the digital zoom function on the laparoscope to avoid the inferior hypogastric nerve plexus in order to preserve urinary function. ${ }^{35,47,63,64} \mathrm{~A}$ key step in the nervesparing approach is that it accurately identifies the anatomic structures in the posterior parametrium prior to removal of the lesions. The surgeon should take the time to distinguish the cranial structure (uterosacral ligaments), the caudad structure (rectovaginal ligaments), and the lateral caudad structure (lateral rectal ligaments). These structures hold the autonomic nerves, which innervate the pelvic viscera. ${ }^{64,65}$ A case series from 2010 found that only three of 16 patients who underwent the nerve-sparing technique needed urinary catheterization that lasted a maximum of 2 days. In comparison, 14 of 55 patients with the classical resection needed catheterization for 3-6 months and two patients had persistent urinary retention. ${ }^{63}$ A prospective cohort study of 61 patients showed longer mean catheter duration of 39.8 days in patients who underwent the nerve-sparing technique, as opposed to a mean catheter duration of 121 days in patients who underwent the classical resection. ${ }^{66}$ Evidently, both analyses were in congruence that the non-nerve-sparing group did not show signs of improvement in their urinary dysfunction after 6 months, and that the nerve-sparing technique was the ideal choice for surgical excision. ${ }^{63,66}$

A recent surgical approach described the use of both laparoscopic and transanal techniques. Laparoscopically, the lesions are shaved and released from the walls of the vagina and surrounding areas. Transanally, a Contour ${ }^{\circledR}$ Transtar $^{\mathrm{TM}}$ stapler (Ethicon Endo-Surgery Inc., Cincinnati, OH, USA) both excises and staples the lesion. The study that addressed this technique was limited in size, only reporting the data 
from six patients. ${ }^{67}$ Four of the patients did not experience any postoperative digestive pain and had clear margins on excision. The technique is very appealing because it avoids opening the lumen and minimizes the leakage of bowel contaminants into the peritoneum. The device also has the ability to excise nodules as high as $10 \mathrm{~cm}$ inward from the anal canal and up to $5 \mathrm{~cm}$ in diameter, as opposed to $7 \mathrm{~cm}$ inward and $2 \mathrm{~cm}$ in diameter, respectively, for other staplers. ${ }^{67}$

Robot-assisted techniques have been reported in recent years. Results from a cohort study conducted over the past 5 years suggest robotics as a useful technique for complex cases such as those concomitant with hysterectomy and sacrocolpopexy. ${ }^{55}$ In a limited study utilizing the robot-assisted approach, out of 19 patients with bowel resection, 23 patients with rectovaginal septum resection, and five bladder resections, one anastomotic leakage was reported. No intraoperative complications or conversion to laparotomy occurred in this study. ${ }^{55}$ More research about this approach and its cost-effectiveness is needed.

\section{Complications and long-term outcomes}

According to a retrospective study conducted with 52 patients who had laparoscopic bowel resections from 2002-2009, only four patients ( $7 \%$ ) had complications. These complications included intra-abdominal bleeding, rectovaginal fistula, and anastomotic leakage. ${ }^{7}$ In a similar retrospective study conducted with 23 patients, three patients (13\%) experienced major complications including anastomotic stenosis, bowel fistula, and bladder fistula. Other complications included constipation in $23 \%$ of patients, dyschezia in $43 \%$ of patients, and dysuria in $18 \%$ of patients. ${ }^{68}$ Rectovaginal fistulas are often seen when lesions are removed in the lower rectum accompanied by a hysterectomy. Using an omental flap or imbricating the seromuscularis layer over the staples can sometimes avoid this complication. ${ }^{47}$ In a comprehensive study of 1,128 full laparoscopic bowel resections for intestinal endometriosis and 19 robot-assisted resections, the overall CR was $8.7 \%$ (94 complications out of 1,147 procedures). The major complications were 24 anastomotic leaks (2.1\%), 18 rectovaginal fistulas (1.5\%), 13 intra-abdominal bleeds (1.1\%), eight pelvic fluid collections $(0.7 \%)$, and two urinary injuries $(0.2 \%)$. The minor complications included nine transient bowel obstructions $(0.8 \%)$, four minor rectal bleedings $(0.3 \%)$, ten wound infections $(0.9 \%)$, two urinary infections $(0.2 \%)$, and four transient urinary retentions $(0.3 \%)$. Recurrence of pain was found in 38 patients. Overall, $99 \%$ of symptoms were alleviated from the surgeries. ${ }^{69}$ Although complications are common, overall improvement in pelvic pain and quality of life is evident with bowel resection. Because some complications are more severe, such as rectal and bladder dysfunctions, patients should be evaluated and counseled carefully before undergoing surgery; various approaches should also be levied according to what optimizes patient satisfaction and outcomes. ${ }^{68} \mathrm{In}$ addition, the recurrence of lesions and symptoms is always a possibility. In patients over 40 years of age with no interest in becoming pregnant, hysterectomy with or without bilateral oophorectomy can be considered. ${ }^{70}$ The surgical approach and the extent of excision should be tailored carefully to the individual patient and to the presenting symptoms.

Quality of life appears to be markedly improved for patients who chose a surgical option. In a study conducted in Brazil, a Short Form-36 Health Status Questionnaire was given to 151 women who underwent laparoscopic resection between 2002 and 2009, and the researchers found significant improvements in pain-related symptoms $(P<0.001)$ and a significant increase in scores across all domains of physical and mental health $(P<0.001) .^{71}$ A cohort study out of Italy used the same Short Form-36 Health Status Questionnaire; the researchers examined quality of life in patients who underwent segmental bowel resection and those who had nodules shaved instead. ${ }^{72}$ Results showed a significant increase in quality of life in all patients, which was congruent with the findings from the aforementioned study. It should be noted, however, that surgical resection did not appear to be superior to shaving. Both methods worked equally well at improving quality of life. Other questionnaires such as the EuroQol Group EQ-5D ${ }^{\mathrm{TM}}$ (EuroQol Group, Rotterdam, The Netherlands) have been used in the analysis of quality of life after laparoscopic resection, and improvements in quality of life were shown to be statistically significant over the course of 3 years of follow up. ${ }^{73}$

In addition to the relief of bowel-related symptoms, sexual dysfunction can be alleviated by surgery. Research has shown that endometriotic nodules in the pouch of Douglas in particular are associated with sexual pain, and laparoscopic resection can significantly improve these symptoms. ${ }^{74}$ It has been shown that 1 year after rectovaginal resection, sexual satisfaction is improved significantly. ${ }^{75,76}$

Surgery can also improve fertility, even when surgery does not remove all endometriotic lesions. ${ }^{77}$ Analyzing whether surgery improves spontaneous conception or IVF is difficult to substantiate. In a systematic review using eleven selected studies, patients with RVE who were infertile before surgery were evaluated for their postsurgical pregnancy rates with spontaneous pregnancy and IVF. The average postoperative conception rate in all women seeking pregnancy apart from preoperative fertility status and IVF performance was 
$39 \%(223 / 571)$, but only $24 \%$ (123/510) in infertile women who tried for spontaneous conception. These data suggest that patients with RVE who undergo surgery should know that postoperative conception is a real possibility, but generic overestimation should be avoided. ${ }^{78}$

\section{Conclusion}

RVE is the most severe form of endometriosis. Symptoms vary among patients, and clinical diagnosis can be challenging. A number of modalities are employed in diagnosis, including physical examination, ultrasound, MRI, colonoscopy, and CT colonography. The gold standard for diagnosis is laparoscopy with histological confirmation. Several studies showed TVS as a very specific, sensitive, affordable, and time-efficient modality for the diagnosis of DIE. However, other modalities, like MRI and CT colonography, can be used and provide information such as the depth at which the lesion infiltrates the bowel wall. Once a diagnosis is determined, medical therapy is usually not adequate to relieve symptoms; although birth control pills are associated with a reduced need for bowel resection. Surgery ranges from superficial partial thickness excisions to radical colorectal resection and reanastomosis. Outcomes from the different surgical options vary; most studies showed that the more radical the resection, the greater the postsurgical CR. However, the recurrence rate was higher with the less aggressive approaches. There has been a significant development of surgical equipment. Research is needed to assess the clinical outcomes associated with using various devices. In conclusion, RVE is a serious diagnosis for patients, and a number of options for the diagnosis and treatment of these patients are available. Diagnostic and therapeutic approaches should be tailored to the individual patient, her symptoms. and her treatment goals.

\section{Disclosure}

The authors report no conflicts of interest in this work.

\section{References}

1. Moawad NS, Guido R, Ramanathan R, Mansuria S, Lee T. Comparison of laparoscopic anterior discoid resection and laparoscopic low anterior resection of deep infiltrating rectosigmoid endometriosis. JSLS. 2011;15(3):331-338.

2. Roberts CP, Rock JA. The current staging system for endometriosis: does it help? Obstet Gynecol Clin North Am. 2003;30(1):115-132.

3. Tarjanne S, Sjöberg J, Heikinheimo O. Rectovaginal endometriosischaracteristics of operative treatment and factors predicting bowel resection. J Minim Invasive Gynecol. 2009;16(3):302-306.

4. Zanetti-Dällenbach R, Bartley J, Müller C, Schneider A, Köhler C. Combined vaginal-laparoscopic-abdominal approach for the surgical treatment of rectovaginal endometriosis with bowel resection: a comparison of this new technique with various established approaches by laparoscopy and laparotomy. Surg Endosc. 2008;22(4):995-1001.
5. Kondo W, Branco AW, Trippia CH, Ribeiro R, Zomer MT. Retrocervical deep infiltrating endometriotic lesions larger than thirty millimeters are associated with an increased rate of ureteral involvement. J Minim Invasive Gynecol. 2013;20(1):100-103.

6. Abrao MS, Gonçalves MO, Dias JA, Podgaec S, Chamie LP, Blasbalg R. Comparison between clinical examination, transvaginal sonography and magnetic resonance imaging for the diagnosis of deep endometriosis. Hum Reprod. 2007;22(12):3092-3097.

7. Jelenc F, Ribič-Pucelj M, Juvan R, Kobal B, Sinkovec J, Salamun V. Laparoscopic rectal resection of deep infiltrating endometriosis. J Laparoendosc Adv Surg Tech A. 2012;22(1):66-69.

8. Sassi S, Bouassida M, Touinsi H, et al. Exceptional cause of bowel obstruction: rectal endometriosis mimicking carcinoma of rectum a case report. Pan Afr Med J. 2011;10:33.

9. Portale TR, Branca A, Scilletta R, Pesce A, Puleo S. Ileo-colic endometriosis: a rare localization of a frequent disease case report. Ann Ital Chir. 2013;84(1).

10. Koninckx PR, Ussia A, Adamyan L, Wattiez A, Donnez J. Deep endometriosis: definition, diagnosis, and treatment. Fertil Steril. 2012;98(3):564-571.

11. Fauconnier A, Fritel X, Chapron C. [Endometriosis and pelvic pain: epidemiological evidence of the relationship and implications]. Gynecol Obstet Fertil. 2009;37(1):57-69. French.

12. Anaf V, Simon P, El Nakadi I, et al. Relationship between endometriotic foci and nerves in rectovaginal endometriotic nodules. Hum Reprod. 2000;15(8):1744-1750.

13. Falcone T, Lebovic DI. Clinical management of endometriosis. Obstet Gynecol. 2011;118(3):691-705.

14. Montanari G, Di Donato N, Benfenati A, et al. Women with deep infiltrating endometriosis: sexual satisfaction, desire, orgasm, and pelvic problem interference with sex. J Sex Med. 2013;10(6):1559-1566.

15. Vercellini P, Somigliana E, Buggio L, Barbara G, Frattaruolo MP, Fedele L. "I can't get no satisfaction": deep dyspareunia and sexual functioning in women with rectovaginal endometriosis. Fertil Steril. 2012;98(6):1503-1511. e1.

16. Ballester M, Oppenheimer A, Mathieu d'Argent E, et al. Deep infiltrating endometriosis is a determinant factor of cumulative pregnancy rate after intracytoplasmic sperm injection/in vitro fertilization cycles in patients with endometriomas. Fertil Steril. 2012;97(2):367-372.

17. Xue Q, Zeng C, Xu Y, et al. [Clinical effect on women with different types of endometriosis related infertility treated by conservative surgery]. Zhonghua Fu Chan Ke Za Zhi. 2013;48(1):16-19. Chinese.

18. Guerrero Hernández A, Oropeza Rechy G, Gómez García E. [Risk factors associated, diagnostic methods and treatment for endometriosis, used in clinical service endometriosis gynecology Hospital General de Mexico (2009-2011)]. Ginecol Obstet Mex. 2012;80(10):637-643. Spanish.

19. Koninckx PR, Martin D. Treatment of deeply infiltrating endometriosis. Curr Opin Obstet Gynecol. 1994;6(3):231-241.

20. Hudelist G, Fritzer N, Thomas A, et al. Diagnostic delay for endometriosis in Austria and Germany: causes and possible consequences. Hum Reprod. 2012;27(12):3412-3416.

21. Dalsgaard T, Hjordt Hansen MV, Hartwell D, Lidegaard O. Reproductive prognosis in daughters of women with and without endometriosis. Hum Reprod. 2013;28(8):2284-2288.

22. Chapron C, Lafay-Pillet MC, Monceau E, et al. Questioning patients about their adolescent history can identify markers associated with deep infiltrating endometriosis. Fertil Steril. 2011;95(3):877-881.

23. Chapron C, Souza C, Borghese B, et al. Oral contraceptives and endometriosis: the past use of oral contraceptives for treating severe primary dysmenorrhea is associated with endometriosis, especially deep infiltrating endometriosis. Hum Reprod. 2011;26(8):2028-2035.

24. Chapron C, Pietin-Vialle C, Borghese B, Davy C, Foulot H, Chopin N. Associated ovarian endometrioma is a marker for greater severity of deeply infiltrating endometriosis. Fertil Steril. 2009;92(2):453-457.

25. Dai Y, Leng JH, Lang JH, et al. [Relationship of pelvic clinic-pathological features and the pain symptoms in ovarian endometrioma]. Zhonghua Fu Chan Ke Za Zhi. 2013;48(2):118-122. Chinese. 
26. Leng JH, Lang JH, Dai Y, Li HJ, Li XY. [Relationship between pain symptoms and clinico-pathological features of pelvic endometriosis]. Zhonghua Fu Chan Ke Za Zhi. 2007;42(3):165-168. Chinese.

27. Roman H, Ness J, Suciu N, et al. Are digestive symptoms in women presenting with pelvic endometriosis specific to lesion localizations? A preliminary prospective study. Hum Reprod. 2012;27(12):3440-3449.

28. Fauconnier A, Chapron C, Dubuisson JB, Vieira M, Dousset B, Bréart G. Relation between pain symptoms and the anatomic location of deep infiltrating endometriosis. Fertil Steril. 2002;78(4):719-726.

29. Cheewadhanaraks S, Peeyananjarassri K, Dhanaworavibul K, Liabsuetrakul T. Positive predictive value of clinical diagnosis of endometriosis. J Med Assoc Thai. 2004;87(7):740-744.

30. Hudelist G, Fritzer N, Staettner S, et al. Uterine sliding sign: a simple sonographic predictor for presence of deep infiltrating endometriosis of the rectum. Ultrasound Obstet Gynecol. 2013;41(6):692-695.

31. Piketty M, Chopin N, Dousset B, et al. Preoperative work-up for patients with deeply infiltrating endometriosis: transvaginal ultrasonography must definitely be the first-line imaging examination. Hum Reprod. 2009;24(3):602-607.

32. Bazot M, Detchev R, Cortez A, Amouyal P, Uzan S, Daraï E. Transvaginal sonography and rectal endoscopic sonography for the assessment of pelvic endometriosis: a preliminary comparison. Hum Reprod. 2003;18(8):1686-1692.

33. Hudelist G, Tuttlies F, Rauter G, Pucher S, Keckstein J. Can transvaginal sonography predict infiltration depth in patients with deep infiltrating endometriosis of the rectum? Hum Reprod. 2009;24(5):1012-1017.

34. Goncalves MO, Podgaec S, Dias JA, Gonzalez M, Abrao MS. Transvaginal ultrasonography with bowel preparation is able to predict the number of lesions and rectosigmoid layers affected in cases of deep endometriosis, defining surgical strategy. Hum Reprod. 2010;25(3): 665-671.

35. Fratelli N, Scioscia M, Bassi E, Musola M, Minelli L, Trivella G. Transvaginal sonography for preoperative assessment of deep endometriosis. J Clin Ultrasound. 2013;41(2):69-75.

36. Saccardi C, Cosmi E, Borghero A, Tregnaghi A, Dessole S, Litta P. Comparison between transvaginal sonography, saline contrast sonovaginography and magnetic resonance imaging in the diagnosis of posterior deep infiltrating endometriosis. Ultrasound Obstet Gynecol. 2012;40(4):464-469.

37. Cosmi E, Saccardi C, Litta P. The sonographic diagnosis of deep endometriosis. J Ultrasound Med. 2009;28(3):410-411.

38. Guerriero S, Ajossa S, Gerada M, D'Aquila M, Piras B, Melis GB. "Tenderness-guided" transvaginal ultrasonography: a new method for the detection of deep endometriosis in patients with chronic pelvic pain. Fertil Steril. 2007;88(5):1293-1297.

39. Reid S, Lu C, Casikar I, et al. Prediction of pouch of Douglas obliteration in women with suspected endometriosis using a new real-time dynamic transvaginal ultrasound technique: the sliding sign. Ultrasound Obstet Gynecol. 2013;41(6):685-691.

40. Rossi L, Palazzo L, Yazbeck C, et al. Can rectal endoscopic sonography predict infiltration depth in patients with deep infiltrating endometriosis of the rectum? Ultrasound Obstet Gynecol. Epub June 10, 2013.

41. Vassilieff M, Suaud O, Collet-Savoye C, et al. [Computed tomographybased virtual colonoscopy: an examination useful for the choice of the surgical management of colorectal endometriosis]. Gynecol Obstet Fertil. 2011;39(6):339-345. French.

42. van der Wat J, Kaplan MD, Roman H, Da Costa C. The use of modified virtual colonoscopy to structure a descriptive imaging classification with implied severity for rectogenital and disseminated endometriosis. J Minim Invasive Gynecol. Epub June 5, 2013.

43. van der Wat J, Kaplan MD. Modified virtual colonoscopy: a noninvasive technique for the diagnosis of rectovaginal septum and deep infiltrating pelvic endometriosis. J Minim Invasive Gynecol. 2007;14(5): 638-643.

44. Bazot M, Darai E, Hourani R, et al. Deep pelvic endometriosis: MR imaging for diagnosis and prediction of extension of disease. Radiology. 2004;232(2):379-389.
45. Stabile Ianora AA, Moschetta M, Lorusso F, et al. Rectosigmoid endometriosis: Comparison between CT water enema and video laparoscopy. Clin Radiol. 2013;68(9):895-901.

46. Krüger K, Behrendt K, Niedobitek-Kreuter G, Koltermann K, Ebert AD. Location-dependent value of pelvic MRI in the preoperative diagnosis of endometriosis. Eur J Obstet Gynecol Reprod Biol. 2013; 169(1):93-98.

47. Pereira RM, Zanatta A, Preti CD, de Paula FJ, da Motta EL, Serafini PC. Should the gynecologist perform laparoscopic bowel resection to treat endometriosis? Results over 7 years in 168 patients. J Minim Invasive Gynecol. 2009;16(4):472-479.

48. Chassang M, Novellas S, Bloch-Marcotte C, et al. Utility of vaginal and rectal contrast medium in MRI for the detection of deep pelvic endometriosis. Eur Radiol. 2010;20(4):1003-1010.

49. Siegelman ES, Oliver ER. MR imaging of endometriosis: ten imaging pearls. Radiographics. 2012;32(6):1675-1691.

50. Gauche Cazalis C, Koskas M, Martin B, Palazzo L, Madelenat P, Yazbeck C. [Preoperative imaging of deeply infiltrating endometriosis in: Transvaginal sonography, rectal endoscopic sonography and magnetic resonance imaging]. Gynecol Obstet Fertil. 2012;40(11):634-641. French.

51. Vercellini P, Somigliana E, Consonni D, Frattaruolo MP, De Giorgi O, Fedele L. Surgical versus medical treatment for endometriosis-associated severe deep dyspareunia: I. Effect on pain during intercourse and patient satisfaction. Hum Reprod. 2012;27(12):3450-3459.

52. Ferrari S, Persico P, DI Puppo F, et al. Continuous low-dose oral contraceptive in the treatment of colorectal endometriosis evaluated by rectal endoscopic ultrasonography. Acta Obstet Gynecol Scand. 2012; 91(6):699-703.

53. Abou-Setta AM, Al-Inany HG, Farquhar CM. Levonorgestrel-releasing intrauterine device (LNG-IUD) for symptomatic endometriosis following surgery. Cochrane Database Syst Rev. 2006;CD005072.

54. De Cicco C, Corona R, Schonman R, Mailova K, Ussia A, Koninckx P. Bowel resection for deep endometriosis: a systematic review. BJOG. 2011;118(3):285-291.

55. Siesto G, Ieda N, Rosati R, Vitobello D. Robotic surgery for deep endometriosis: a paradigm shift. Int J Med Robot. Epub June 13, 2013.

56. Berkes E, Bokor A, Rigó J Jr. [Current treatment of endometriosis with laparoscopic surgery]. Orv Hetil. 2010;151(28):1137-1144. Hungarian.

57. Hidaka T, Nakashima A, Hashimoto Y, Saito S. Effects of laparoscopic radical surgery for deep endometriosis on endometriosis-related pelvic pain. Minim Invasive Ther Allied Technol. 2012;21(5):355-361.

58. Robbins ML. Excision of endometriosis with laparosonic coagulating shears. J Am Assoc Gynecol Laparosc. 1999;6(2):199-203.

59. Kristensen J, Kjer JJ. Laparoscopic laser resection of rectovaginal pouch and rectovaginal septum endometriosis: the impact on pelvic pain and quality of life. Acta Obstet Gynecol Scand. 2007;86(12):1467-1471.

60. Meuleman C, Tomassetti C, Wolthuis A, et al. Clinical outcome after radical excision of moderate-severe endometriosis with or without bowel resection and reanastomosis: a prospective cohort study. Ann Surg. Epub April 10, 2013.

61. Meuleman C, Tomassetti C, D'Hooghe TM. Clinical outcome after laparoscopic radical excision of endometriosis and laparoscopic segmental bowel resection. Curr Opin Obstet Gynecol. 2012;24(4): 245-252.

62. Roman H, Vassilieff M, Tuech JJ, et al. Postoperative digestive function after radical versus conservative surgical philosophy for deep endometriosis infiltrating the rectum. Fertil Steril. 2013;99(6):1695-1704.

63. Kavallaris A, Banz C, Chalvatzas N, et al. Laparoscopic nerve-sparing surgery of deep infiltrating endometriosis: description of the technique and patients' outcome. Arch Gynecol Obstet. 2011;284(1):131-135.

64. Ceccaroni M, Clarizia R, Roviglione G, Ruffo G. Neuro-anatomy of the posterior parametrium and surgical considerations for a nerve-sparing approach in radical pelvic surgery. Surg Endosc. Epub June 20, 2013. 
65. Azaïs H, Collinet P, Delmas V, Rubod C. [Uterosacral ligament and hypogastric nerve anatomical relationship. Application to deep endometriotic nodules surgery]. Gynecol Obstet Fertil. 2013;41(3): 179-183. French.

66. Ceccaroni M, Clarizia R, Bruni F, et al. Nerve-sparing laparoscopic eradication of deep endometriosis with segmental rectal and parametrial resection: the Negrar method. A single-center, prospective, clinical trial. Surg Endosc. 2012;26(7):2029-2045.

67. Bridoux V, Roman H, Kianifard B, et al. Combined transanal and laparoscopic approach for the treatment of deep endometriosis infiltrating the rectum. Hum Reprod. 2012;27(2):418-426.

68. Boileau L, Laporte S, Bourgaux JF, et al. [Laparoscopic colorectal resection for deep pelvic endometriosis: Evaluation of post-operative outcome]. J Gynecol Obstet Biol Reprod (Paris). 2012;41(2): 128-135. French.

69. Ruffo G, Rossini R. The outcomes of laparoscopic resection of bowel endometriosis. Curr Opin Obstet Gynecol. 2013;25(4):302-307.

70. Borghese B, Santulli P, Streuli I, Lafay-Pillet MC, de Ziegler D, Chapron C. [Recurrence of pain after surgery for deeply infiltrating endometriosis: How does it happen? How to manage?]. J Gynecol Obstet Biol Reprod (Paris). 2012. French.

71. Bassi MA, Podgaec S, Dias JA, D’Amico Filho N, Petta CA, Abrao MS. Quality of life after segmental resection of the rectosigmoid by laparoscopy in patients with deep infiltrating endometriosis with bowel involvement. J Minim Invasive Gynecol. 2011;18(6):730-733.
72. Mabrouk M, Montanari G, Guerrini M, et al. Does laparoscopic management of deep infiltrating endometriosis improve quality of life? A prospective study. Health Qual Life Outcomes. 2011;9:98.

73. Roman JD. Surgical treatment of endometriosis in private practice: cohort study with mean follow-up of 3 years. JMinim Invasive Gynecol. 2010;17(1):42-46.

74. Anaf V, Simon P, El Nakadi I, Simonart T, Noel J, Buxant F. Impact of surgical resection of rectovaginal pouch of douglas endometriotic nodules on pelvic pain and some elements of patients' sex life. J Am Assoc Gynecol Laparosc. 2001;8(1):55-60.

75. Kössi J, Setälä M, Mäkinen J, Härkki P, Luostarinen M. Quality of life and sexual function 1 year after laparoscopic rectosigmoid resection for endometriosis. Colorectal Dis. 2013;15(1):102-108.

76. Setälä M, Härkki P, Matomäki J, Mäkinen J, Kössi J. Sexual functioning, quality of life and pelvic pain 12 months after endometriosis surgery including vaginal resection. Acta Obstet Gynecol Scand. 2012;91(6): 692-698.

77. Donnez J, Nisolle M. Advanced laparoscopic surgery for the removal of rectovaginal septum endometriotic or adenomyotic nodules. Baillieres Clin Obstet Gynaecol. 1995;9(4):769-774.

78. Vercellini P, Barbara G, Buggio L, Frattaruolo MP, Somigliana E, Fedele L. Effect of patient selection on estimate of reproductive success after surgery for rectovaginal endometriosis: literature review. Reprod Biomed Online. 2012;24(4):389-395.
International Journal of Women's Health

\section{Publish your work in this journal}

The International Journal of Women's Health is an international, peerreviewed open-access journal publishing original research, reports, editorials, reviews and commentaries on all aspects of women's healthcare including gynecology, obstetrics, and breast cancer. The manuscript management system is completely online and includes

\section{Dovepress}

a very quick and fair peer-review system, which is all easy to use. Visit http://www.dovepress.com/testimonials.php to read real quotes from published authors. 